\title{
APPROXIMATELY CONTINUOUS TRANSFORMATIONS
}

\section{CASPER GOFFMAN AND DANIEL WATERMAN}

1. An interesting class of real functions of a single real variable, the approximately continuous functions, was introduced by Denjoy [1] in his work on derivatives. The two principal facts discovered by Denjoy are that these functions are of Baire class 1 and have the Darboux property. Ridder [2] showed that the arguments of Denjoy apply to real functions of $n$ variables.

In this paper we discuss approximately continuous transformations from euclidean spaces into arbitrary metric spaces. We show that the image under such a transformation is always separable, that the transformation is of Baire class 1, and that it has a Darboux property.

In $\$ 2$ the Darboux property for real functions of a real variable is discussed. In $\S 3$ the notions of approximate continuity and metric density are defined in our context. In $\$ 4$ it is shown that the image of $E_{n}$ under an approximately continuous transformation is separable and such transformations are of Baire class 1 . In $\$ 5$ we introduce the notion of homogeneity of sets relative to metric density. $E_{n}$ may be topologized by taking the homogeneous sets as open sets. ${ }^{1}$ We show that the open connected subsets of $E_{n}$ are connected in this topology. The approximately continuous transformations are the continuous transformations in the new topology. In $\S 6$, we define $d$-regular sets, closed sets with connected interior and certain boundary restrictions. It is shown that approximately continuous transformations take $d$ regular sets into connected sets, i.e., they have a Darboux property. Further remarks on connectivity are made in $\$ 7$.

2. A real function $f$, defined on an open interval $I$, has the Darboux property if, for every $\alpha, \beta \in I, \alpha<\beta$, the image $f((\alpha, \beta))$ of $(\alpha, \beta)$ contains the open interval whose end points are $f(\alpha)$ and $f(\beta)$.

An equivalent statement is that $f$ takes every connected set into a connected set. This clearly implies the Darboux property. Conversely, suppose $f$ has the Darboux property. If $S$ is a connected subset of $I$, then $S$ is an interval (perhaps degenerate). Suppose the image $f(S)$ of $S$ is not connected, i.e., it is not an interval. There exist $c, d$, and $\eta$ with $c<\eta<d$, such that $c, d \in f(S)$ and $\eta \notin f(S)$. Then the image of the open interval with endpoints $f^{-1}(c)$ and $f^{-1}(d)$ does not contain $(c, d)$.

Received by the editors April 5, 1960.

${ }^{1}$ We should like to express our appreciation to M. Henriksen and D. G. Johnson for valuable discussions regarding this topology. 
Another condition equivalent to the Darboux property is that $f$ takes closed intervals into connected sets. It is interesting, however, that the condition that $f$ takes open intervals into connected sets is not equivalent to the Darboux property. The equivalence of the first of these two conditions is an immediate consequence of the fact that every connected set is the union of an increasing sequence of closed intervals. Clearly, every function which has the Darboux property also satisfies the second of these two conditions. Consider, however, the function $f$ defined on the open interval $(-1,1)$ by

$$
f(x)=\begin{array}{cl}
0, & x<0 \\
1, & x=0 \\
\sin (1 / x), & x>0 .
\end{array}
$$

It is clear that this function takes open intervals into connected sets but does not have the Darboux property.

3. Let $S$ be a measurable set in euclidean $n$-space. The upper metric density of $S$ at a point $p$ is

$$
\lim _{n \rightarrow \infty} \sup _{Q}\left[\frac{m(S \cap Q)}{m(Q)}: m(Q)<1 / n\right]
$$

where $Q$ is an open $n$-cube containing $p$. The lower metric density is defined similarly. If they are equal, the common value is called the metric density of $S$ at $p$.

A transformation $f$ from a euclidean space to a topological space is said to be approximately continuous at a point $p$ if, for every open set $G$ containing $f(p)$, the set $f^{-1}(G)$ has metric density 1 at $p$.

4. Let $f$ be an approximately continuous transformation from euclidean $n$-space $E_{n}$ to a metric space $S$. We prove

TheOREM 1. The set $f\left(E_{n}\right)$ is a separable subspace of $S$.

Proof. Let $\epsilon>0$. For every $m$, let $K_{m}$ be the closed sphere in $E_{n}$ with the origin as center and with radius equal to $m$. Every $p \in K_{m}$ is contained in a sequence $\left\{Q_{p, i}\right\}, i=1,2, \cdots$, of open cubes with measures converging to zero, and such that the set of points $q \in Q_{p, i}$ for which $d(f(p), f(q))<\epsilon$ has relative measure greater than $1-\epsilon$ in $Q_{p, i \text {. }}$ The set of cubes $\left[Q_{p, i}: p \in K_{m}, i=1,2, \cdots\right]$ covers $K_{m}$ in the sense of Vitali. Hence there is a countable subset $\left\{Q_{p_{k}, i_{k}}\right\}$ of pairwise disjoint cubes which covers all of $K_{m}$ except for a set of measure zero. Thus there is a countable subset $D_{m, \mathrm{e}}$ of $S$ such that for all $q \in K_{m}$, except for a set of measure less than $\epsilon m\left(K_{m}\right), f(q)$ has distance 
less than $\epsilon$ from $D_{m, \epsilon}$. Let $\epsilon_{\nu}$ be a sequence of positive numbers converging to zero. There is a set $Z \subset E_{n}$ of measure zero such that $D=\bigcup_{m, \nu=1}^{\infty} D_{m, \epsilon_{\nu}}$ is dense in $f\left(E_{n}-Z\right)$. Now let $p \in Z$ and $\delta>0$. By the approximate continuity of $f$ at $p$, there is a $q \in E_{n}-Z$ such that $d(f(p), f(q))<\delta / 2$. There is a $\zeta \in D$ such that $d(f(q), \zeta)<\delta / 2$ and so $d(f(p), \zeta)<\delta$. Thus $D$ is dense in $f\left(E_{n}\right)$ and $f\left(E_{n}\right)$ is separable.

We now show that if $f$ is an approximately continuous transformation from $E_{n}$ to a metric space $S$, it is of Baire class 1 .

Suppose $f$ is not of Baire class 1 . Then there is a nonempty perfect set $R$ such that, at every point of $R, f$ is discontinuous relative to $R$. $R$ is a complete metric space. For every $m$, let $R_{m}$ be the subset of $R$ at which the saltus of $f$ is not less than $1 / \mathrm{m}$. At least one $R_{m}$ contains an open sphere $K$ in $R$. Then the saltus of $f$ relative to $K$ is not less than $2 \alpha=1 / m$ at every point of $K$. Since the closure of any open subsphere of $K$ is perfect, the space $K$ is of the second category.

Cover $f\left(E_{n}\right)$ by a countable set of open spheres of radius $\alpha / 3$. The intersection of $f\left(E_{n}\right)$ with one of these, having center $\zeta$, has inverse $T_{1}$ dense in a perfect subset $P \subset K$. Let $T_{2}$ be the set of $p \in E_{n}$ for which $d(f(p), \zeta)>2 \alpha / 3$. Then $T_{2}$ is also dense in $P$ since the saltus of $f$ relative to $P$ is not less than $2 \alpha$ at every point of $P$ and $T_{1}$ is dense in $P$.

Let $\left\{p_{n}\right\}$ be a countable dense subset of $T_{1}$ which is dense in $P$ and let $\left\{\epsilon_{n}\right\}$ be any sequence of positive numbers converging to zero. Since $T_{1}$ has density one at each of its points, there is a sequence of open cubes, $\left\{Q_{n}\right\}, p_{n} \in Q_{n}, \lim _{n \rightarrow \infty} m\left(Q_{n}\right)=0$, such that the relative measure of $T_{1}$ in $Q_{n}$ exceeds $1-\epsilon_{n}$. The set $V_{1}$ of points belonging to infinitely many $Q_{n}$ is residual relative to $P$. In the same fashion we can construct another residual set $V_{2}$ corresponding to $T_{2}$.

The set $V=V_{1} \cap V_{2}$ is residual relative to $P$. The upper metric densities of $T_{1}$ and $T_{2}$ are equal to one at every point of $V$. Thus for any $p \in V$ we have simultaneously

$$
d(f(p), \zeta) \leqq \alpha / 3 \text { and } d(f(p), \zeta) \geqq 2 \alpha / 3,
$$

and so $V$ is empty. This contradiction establishes

THEOREM 2. An approximately continuous transformation from a euclidean space to a metric space is of Baire class 1.

5. A measurable set in euclidean $n$-space will be called homogeneous if its density is one at each of its points. Clearly the union or finite intersection of homogeneous sets is homogeneous. Since the empty set and the whole space are homogeneous, $E_{n}$ can be topologized by taking the homogeneous sets as open sets. This topology is evidently 
Hausdorff. Most important for us is that the approximately continuous transformations are the continuous transformations in this topology, which we will refer to as the $d$-topology.

Clearly $p$ is a limit point of a set $S$ in the $d$-topology if and only if $S$ has positive upper metric density at $p$. A point with this property will be called a d-limit point. A set which is connected in the $d$-topology, i.e., $d$-connected, is then one which is not the union of two nonempty subsets neither of which contains a $d$-limit point of the other.

From the above remarks it follows immediately that approximately continuous transformations take $d$-connected sets into connected sets.

Denjoy observed that an open interval is not the union of two disjoint, nonempty, homogeneous sets. Clearly, for homogeneous sets, $d$-connectedness and Denjoy's property are equivalent. The proof of Denjoy depends on properties of derivatives and cannot be extended to our case. The result, however, is still valid.

\section{THEOREM 3. Every open connected subset of $E_{n}$ is d-connected.}

Proof. Suppose $S$ is open and connected and there exist sets $A$ and $B$, nonempty, disjoint, homogeneous, and such that $A \cup B=S$. Let $p_{0} \in A, p_{1} \in B$, and $C$ be a simple arc in $S$ connecting $p_{0}$ and $p_{1}$.

We will say that a cube is oriented if its edges are parallel to those of a given cube. For every point $p$ and $r>0$, let $Q(p, r)$ be the oriented closed cube of center $p$ and edge $r$. For any measurable set in $E_{n}$, the relative measure of the set in $Q(p, r)$ is a continuous function of $p$ for fixed $r$.

Choose $r$ so that $Q(p, r) \subset S$ for every $p \in C$, and so that the relative measure of $A$ in $Q(p, r)$ exceeds $1 / 2$ for $p=p_{0}$ and is less than $1 / 2$ for $p=p_{1}$. This is possible since $A$ has density one at $p_{0}$ and density zero at $p_{1}$. There must then be a point $\not \in C$ such that the relative

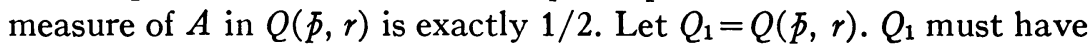
interior points $p_{0}^{1} \in A, p_{1}^{1} \in B$. Join these by a line segment $C^{1}$. We may choose $r^{1}<r / 2$ so that $Q\left(p, r^{1}\right) \subset Q_{1}$ for every $p \in C^{1}$ and so that the relative measure of $A$ in $Q\left(p, r^{1}\right)$ exceeds $1 / 2$ for $p=p_{0}^{1}$ and is less than $1 / 2$ for $p=p_{1}^{1}$. There is then a point $p^{*} \in C^{1}$ such that the relative measure of $A$ in $Q\left(p^{*}, r^{1}\right)$ is exactly $1 / 2$. Let $Q_{2}=Q\left(p^{*}, r^{1}\right)$. Continuing this process, we construct a nested sequence $\left\{Q_{n}\right\}$ of closed oriented cubes in each of which the relative measure of $A$ is $1 / 2$. This sequence converges to a point $p^{\prime} \in S$, but neither $A$ nor $B$ can have metric density one at $p^{\prime}$. Since $A$ and $B$ are homogeneous, $p^{\prime} \notin A, p^{\prime} \notin B$. This contradiction establishes the theorem.

6. Consider a closed set, with connected interior, whose boundary 
points are $d$-limit points of the interior. Such a set will be called $d$-regular. Closed convex sets are examples of $d$-regular sets. It should be noted that the addition of a sharp spine to the boundary of such a set suffices to destroy $d$-regularity.

We will show that $d$-regular sets are $d$-connected, which will at once give a generalization of Denjoy's result on the Darboux property.

Theorem 4. An approximately continuous transformation from a euclidean space to a metric space takes d-regular sets into connected sets.

Proof. Suppose that a $d$-regular set $S$ is not $d$-connected. Then $\mathrm{S}=A \cup B$ such that $A$ and $B$ are disjoint, nonempty, and each contains none of the other's $d$-limit points. One of the sets, say $A$, must then be contained entirely in the boundary of $S$, for otherwise this separation would induce a separation of the interior of $S$ which is contrary to Theorem 3 . Then $A$ contains a $d$-limit point of the interior of $S$ and hence of $B$, which is contrary to our assumption.

7. We conclude with a few additional remarks concerning the $d$ topology. Although this topology is Hausdorff, it is not Lindelöf and is evidently quite pathological in other respects. We will confine ourselves to some observations concerning connectedness.

There are, of course, strictly more connected sets under the ordinary topology of $E_{n}(n>1)$ than under the $d$-topology since the latter is finer and line segments are not $d$-connected. By Theorem 3 , an open connected set is $d$-connected. However, there are more open connected sets under the $d$-topology.

Consider the open unit sphere in $E_{2}$. Delete an open radius. The resulting set is open in the $d$-topology since the origin is a point at which the set has density one and is, therefore, interior. We show that the set is $d$-connected.

Suppose it is the union of two disjoint, nonempty, homogeneous sets $A$ and $B$. Let $p_{0} \in A, p_{1} \in B$. We now connect these points with a curve $C$ as in the proof of Theorem 3. Proceeding as in that proof, we produce a point $p$ at which the density $A$ nor $B$ is one. This contradiction establishes the $d$-connectedness of the set.

We next consider the complement in $E_{n}$ of any countable set. This set, which we denote by $R$, is $d$-connected.

We suppose that $R$ may be separated into sets $A$ and $B$ as described above. Choose $\alpha_{1}, \alpha_{2} \in A, \beta_{1}, \beta_{2} \in B$. Connect $\alpha_{1}$ and $\beta_{1}$ with a simple closed curve $C_{1}$, and $\alpha_{2}$ and $\beta_{2}$ with $C_{2}$ where $C_{1}$ and $C_{2}$ are in $R$ and do not intersect. Proceed on each of these, as in Theorem 3, to locate a point and a small closed cube about it such that $A$ has relative 
measure $1 / 2$ in the cube. The cubes may be chosen disjoint. In each of these cubes we choose 2 points of $A$ and 2 points of $B$ and repeat the operation. At the $n$th stage we will have produced $2^{n}$ closed cubes in which $A$ has relative measure $1 / 2$. The cubes are chosen, as in the proof of Theorem 3, so that the edge tends to zero. The cubes tend, then, to $c$ points at each of which the density of neither $A$ nor $B$ is one. Since $R$ contains all of $E_{n}$ except for a countable set, $R$ contains points at which the metric density of neither $A$ nor $B$ is one. Hence this separation is not possible and $R$ is $d$-connected.

\section{REFERENCES}

1. A. Denjoy, Sur les fonctions dérivées sommables, Bull. Soc. Math. France vol. 43 (1916) pp. 161-248.

2. J. Ridder, Über approximativ stetige Funktionen von zwei (und mehreren) Veranderlichen, Fund. Math. vol. 13 (1927) pp. 201-209.

Purdue University AND

UNIVERSITY OF Wisconsin, MiLWAUKEE 